\title{
Communication \\ A Wideband Cryogenic Readout Amplifier with Temperature-Insensitive Gain for SNSPD
}

\author{
Xiaokang Niu ${ }^{1}$, Lianming $\mathrm{Li}^{1,2}{ }^{-}, \mathrm{Xu} \mathrm{Wu}^{1,2} \mathbb{D}$ and Dongming Wang ${ }^{1,2, *}$ \\ 1 National Mobile Communications Research Laboratory, Southeast University, Nanjing 210000, China; \\ niuxiaokang@seu.edu.cn (X.N.); lianming.li@seu.edu.cn (L.L.); xu.wu@seu.edu.cn (X.W.) \\ 2 Purple Mountain Laboratory, Nanjing 210000, China \\ * Correspondence: wangdm@seu.edu.cn
}

check for

updates

Citation: Niu, X.; Li, L.; Wu, X.; Wang, D. A Wideband Cryogenic Readout Amplifier with Temperature-Insensitive Gain for SNSPD. Sensors 2022, 22, 1225. https://doi.org/10.3390/s22031225

Academic Editors:

Ivano Ruo-Berchera, Paolo Traina and Thomas P. Karnowski

Received: 28 November 2021

Accepted: 2 February 2022

Published: 6 February 2022

Publisher's Note: MDPI stays neutral with regard to jurisdictional claims in published maps and institutional affiliations.

Copyright: (C) 2022 by the authors. Licensee MDPI, Basel, Switzerland. This article is an open access article distributed under the terms and conditions of the Creative Commons Attribution (CC BY) license (https:// creativecommons.org/licenses/by/ $4.0 /$ )

\begin{abstract}
This paper presents a temperature-insensitive wideband cryogenic amplifier for superconducting nanowire single-photon detectors (SNSPD). With a proposed folded diode-connected transistor load to realize a good device-tracking feature, the theoretical derivations the simulations and test results prove that the amplifier-gain cell has a stable gain performance over a wide temperature range, solving the issues of a lack of the accurate cryogenic device models. The amplifier achieves a gain of $26 \mathrm{~dB}$ from $100 \mathrm{kHz}$ to $1 \mathrm{GHz}$ at $4.2 \mathrm{~K}$, consuming only $1.8 \mathrm{~mW}$ from a $1.8 \mathrm{~V}$ supply. With a $0.13-\mu \mathrm{m}$ SiGe BiCMOS process, the chip area is $0.5 \mathrm{~mm}^{2}$.
\end{abstract}

Keywords: cryogenic amplifier; superconducting nanowire single photon detectors; SNSPD; wideband; readout; low power

\section{Introduction}

As key enabling components, SNSPDs [1,2] play an important role in the applications of high-speed quantum key distribution (QKD) [3,4], light detection and ranging (LIDAR) [5], high-sensitivity bio-medical imaging [6-9], remote sensing and detection [10]. To detect weak electrical SNSPD output signals [11], a readout amplifier with high gain and low noise performance is needed. As the traditional readout amplifiers have relatively large sizes and high-power consumption, they are difficult to assemble with SNSPDs. For illustration, as shown in Figure 1, the readout amplifier works at room temperature, and it is connected to the SNSPD in the cryogenic environment with a long coaxial cable. With tests, on one hand it is found that the long coaxial cable limits the size of the SNSPD readout system, making it difficult to realize compact multi-element SNSPD arrays. On the other hand, this large readout system would deteriorate the signal quality and introduce large signal jitters.

Considering the above issues, it is preferable to place the readout amplifier in a cryogenic environment, resulting in a compact cryogenic SNSPD readout system, as shown in Figure 2. In this way, the interconnecting line between the SNSPD and the cryogenic readout amplifier can be reduced substantially, reducing the insertion loss and signal interference. From the implementation perspective, due to the limited cooling capacity of the cryogenic refrigerator, the power consumption of the cryogenic amplifier should be low enough. The traditional radio frequency (RF) amplifiers with topologies such as Doherty and balanced structures exhibit remarkable performance in terms of power efficiency, gain, bandwidth and linearity [12,13], but they are not specifically designed for cryogenic applications. Different from these amplifiers, the cryogenic amplifier prefers higher input impedance, instead of conventional $50 \mathrm{ohm}$ impedance, to obtain higher SNSPD signal voltage amplitude for a better signal-to-noise ratio [14,15]. Recently, several cryogenic readout amplifiers have been realized using III-V and silicon processes. A HEMT $0.5 \sim 13 \mathrm{GHz}$ low noise amplifier with a gain of $38 \sim 44 \mathrm{~dB}$ is presented in [16]. However, this amplifier dissipates a high power consumption of $15 \mathrm{~mW}$, making it difficult to be placed 
in the cryogenic environment. In $[17,18]$, the power consumption of the realized amplifiers is lower than $1 \mathrm{~mW}$, but their working frequencies are $4 \sim 8 \mathrm{GHz}$, which is not feasible for SNSPD readout applications. Note that compared with the SiGe process, the III-V process has the disadvantage of a low integration level and high cost. In [19], based on the CherryHooper amplifier topology, a wideband cryogenic amplifier is implemented with the SiGe process, demonstrating a bandwidth of $3.4 \mathrm{GHz}$, with a power consumption of $4.3 \mathrm{~mW}$. However, due to the high sensitivity of bipolar transistor transconductance to the operating temperature and the lack of accurate cryogenic device models, the cryogenic amplifier gain performance cannot be predicated easily in the design and simulation, resulting in the need for additional biasing tuning to assure the proper working of the amplifier.

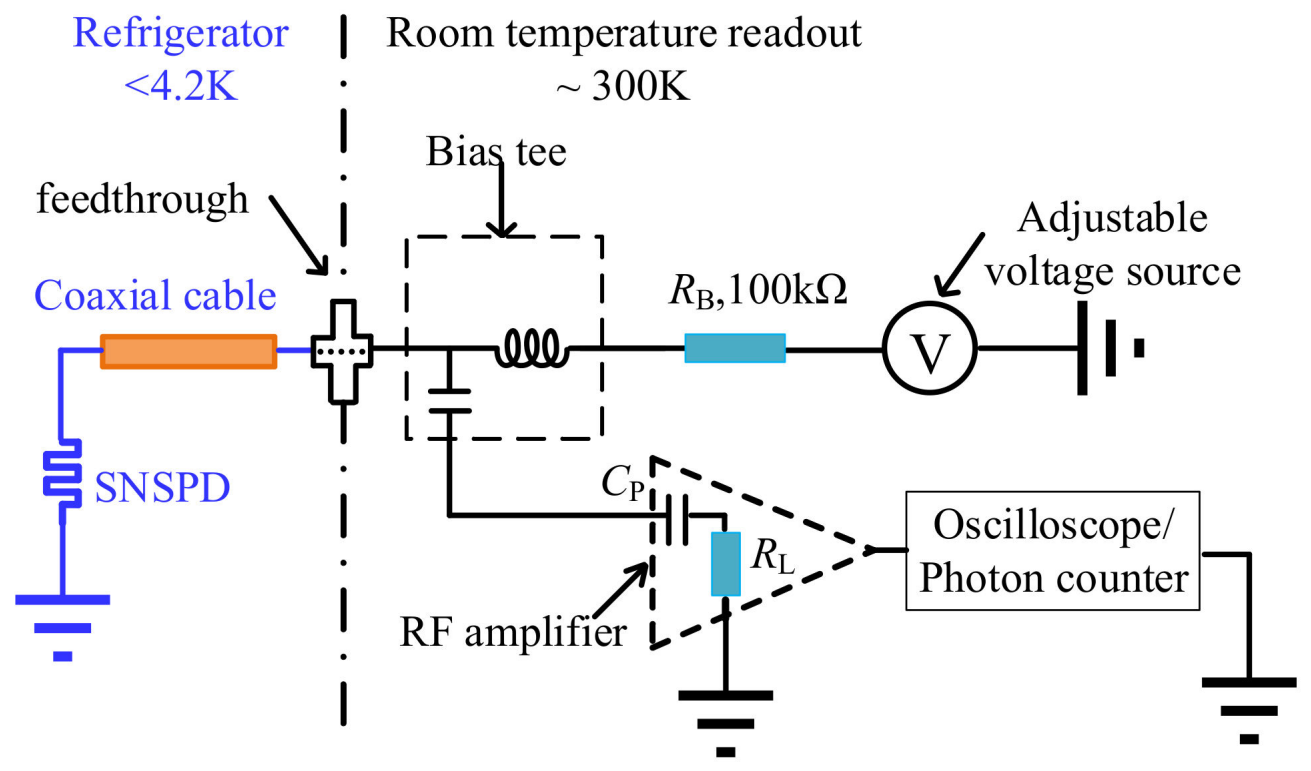

Figure 1. The traditional SNSPD readout scheme.

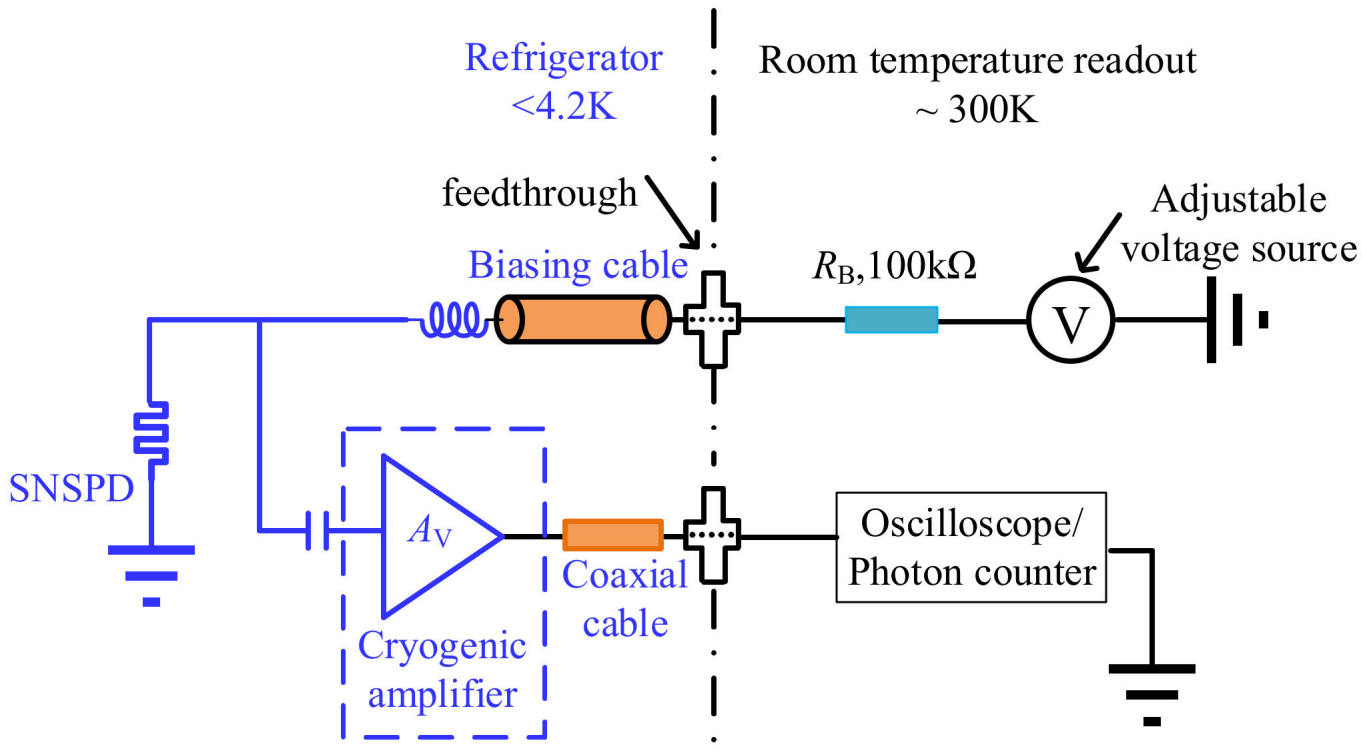

Figure 2. The cryogenic SNSPD readout scheme.

To address the above issues, this paper proposes a robust temperature-insensitive gain-cell structure with a folded diode-connected transistor load. With calculations and 
simulations, it is proved that the gain cell has a stable gain across a wide temperature range due to its good device-tracking feature. With a $0.13 \mu \mathrm{m} \mathrm{SiGe} \mathrm{BiCMOS} \mathrm{process,} \mathrm{the} \mathrm{test}$ results show that the amplifier achieved a $26 \mathrm{~dB}$ gain from $100 \mathrm{kHz}$ to $1 \mathrm{GHz}$ at $4.2 \mathrm{~K}$, while consuming only $1.8 \mathrm{~mW}$ without complex biasing tuning.

The paper is organized as follows. Section 2 presents the amplifier requirements and topology. Section 3 explains the design detail of the temperature-insensitive gain cell. The circuit implementation, simulation and measurement results are described in Section 4. Finally, a conclusion is provided in Section 5.

\section{The Amplifier Requirements and Topology}

For the proper design of the readout amplifier, it is important to evaluate the SNSPD readout requirements. For illustration, Figure 3a shows that the SNSPD is AC-coupled to the amplifier using a capacitor. With a current bias, typically the working behavior of the SNSPD can be described with an equivalent electrical lumped model. As shown in Figure 3a, this lumped model consists of a kinetic inductor $L_{\mathrm{K}}$, a resistor $R_{\mathrm{N}}$ and a switch [20]. To establish a proper current loop and to quench the device, a parallel resistor $R_{P}$ with $50 \mathrm{Ohm}$ is added [21]. Note that as the kinetic inductor $L_{\mathrm{K}}$ is much larger than its Faraday inductance and the parasitic capacitance is relatively small, the parasitic capacitance is ignored without losing the model accuracy, and the time constant of the transient pulse is mainly determined by the kinetic inductor $L_{\mathrm{K}}$ and corresponding resistance. When the SNSPD is in the superconducting state, the switch is closed. In contrast, when an incident photon is absorbed by the SNSPD, the switch is opened, pushing the current into the parallel resistor $R_{P}$. Accordingly, a pulse voltage signal, typically with several hundred microvolts amplitude, is generated [22], as shown in Figure 3b. Normally, its rising time is around 200 ps, while its falling time ranges from 10 to 60 ns. To amplify such a pulse signal with high fidelity, the amplifier bandwidth should be wide enough. Otherwise the signal edge performance will be degraded, introducing several non-idealities and even leading to a wrong detection.

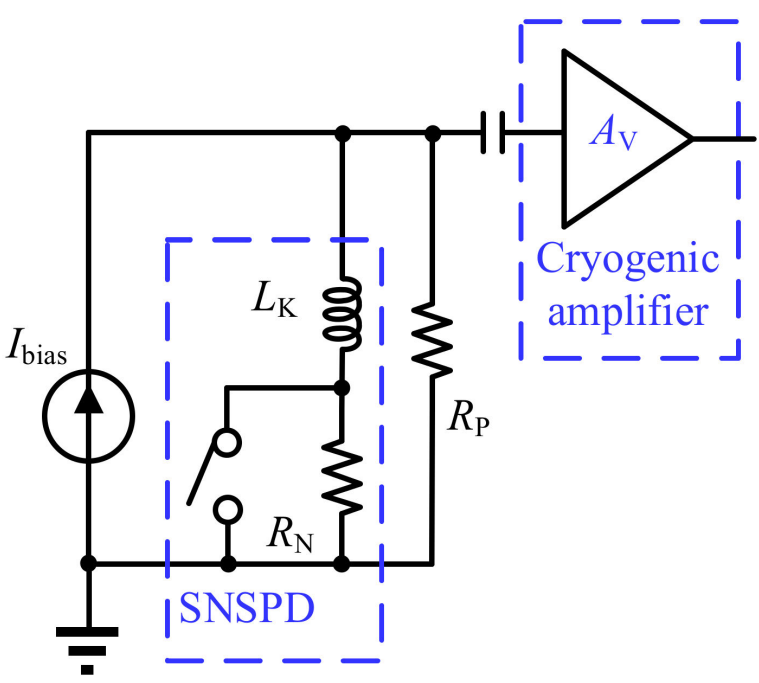

(a)

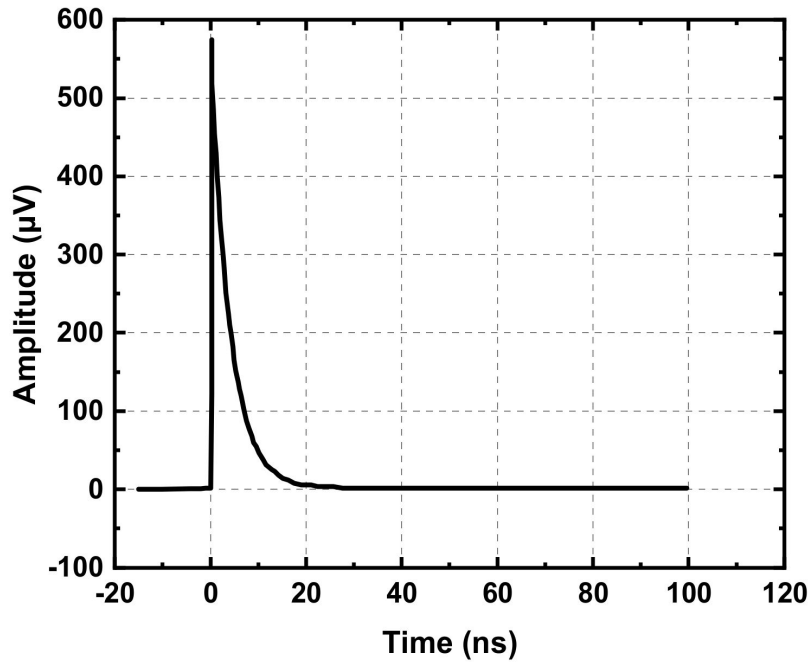

(b)

Figure 3. The connection of the SNSPD and readout amplifier, and output signal: (a) The SNSPD model and its connection with the amplifier; (b) Typical SNSPD output pulse signal.

To evaluate the bandwidth requirement of the amplifier, with an ideal unity gain amplifier, the following simulations were undertaken with different bandwidth conditions. As shown in Figure $4 a, b$, the solid lines represent the SNSPD input pulse signal with a 200 ps rising time and $20 \mathrm{~ns}$ falling time, while the dashed lines are the output signals after passing through the unity gain amplifier. As indicated, when the amplifier bandwidth 
is $100 \mathrm{MHz}$, the output signal has a significant delay of about $20 \mathrm{~ns}$ and experiences ringing issues. As shown in Figure $4 \mathrm{~b}$, when the bandwidth is increased to $1 \mathrm{GHz}$, the output agrees well with the input signal, with a delay of only $1.5 \mathrm{~ns}$, which is smaller than $1 / 10$ pulse width, meeting the system requirement. Note that a larger bandwidth is better for the signal fidelity, but it would introduce more noise and the amplifier dissipates more power. Considering these design trade-offs, in this work, the high cut-off frequency of the amplifier is set to around $1 \mathrm{GHz}$. Moreover, considering the issues of metastability, noise, etc., the minimum input signal of a high speed comparator needs to be higher than $5 \mathrm{mV}$ [23] for a good BER (bit error rate) performance. With a typical amplitude of a SNSPD output signal of about $600-\mu \mathrm{V}$, the gain of the readout amplifier should be larger than $20 \mathrm{~dB}[19,24,25]$, thereby assuring the output signal swing is large enough for the post ADC processing circuitry.

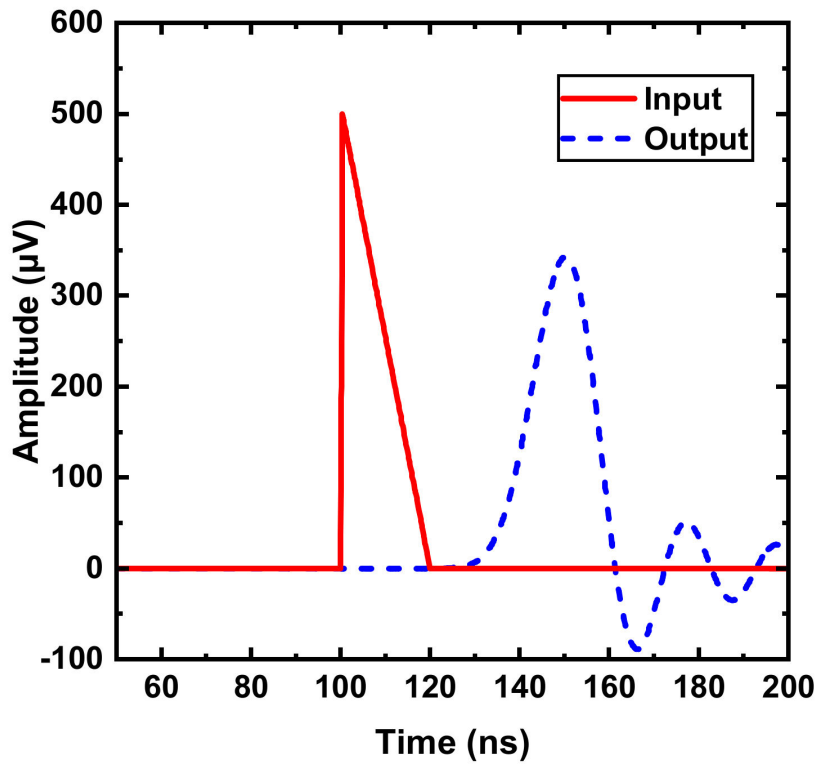

(a)

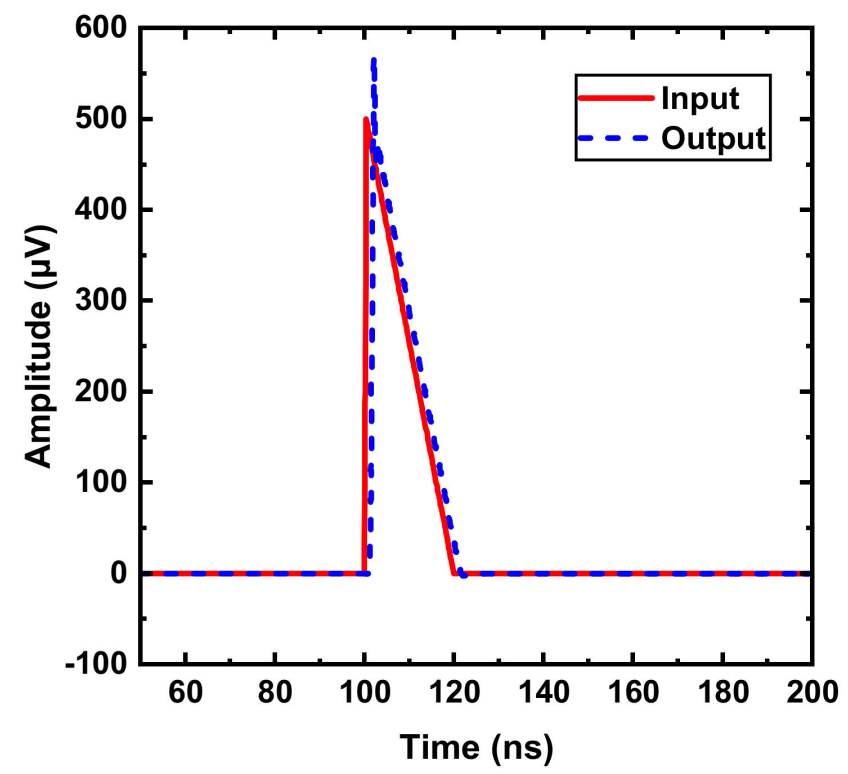

(b)

Figure 4. Transient response of the signal with different bandwidths: (a) Bandwidth $=100 \mathrm{MHz}$; (b) Bandwidth $=1 \mathrm{GHz}$.

To achieve sufficient gain performance, in this paper, a 4-stage cryogenic amplifier is proposed, as each stage has a limited gain. Figure 5a shows the topology of the proposed wideband bipolar cryogenic amplifier, which consists of three temperature-insensitive gain cells and an output buffer stage, shown in Figure $5 b, c$, respectively. Note that, the differential structures are used in these gain cells to improve the common-mode rejection ratio, making the amplifier robust to interference.

As indicated in Figure 5a, its input gain cell is a kind of pseudo-differential configuration. By terminating the parallel resistor $R_{\mathrm{b} 2}$ and capacitor $C_{1}$ to ground, the single-ended to differential signal conversion is realized. To achieve better large signal performance and a better driving capability, an emitter degeneration structure is employed in the output buffer stage to drive the standard $50 \mathrm{Ohm}$ impedance of the measurement equipment. Considering the lack of an accurate cryogenic device model, all the gain cells and the output buffer are AC-coupled to increase the DC biasing flexibility and to remove the DC offset from differential signals. 


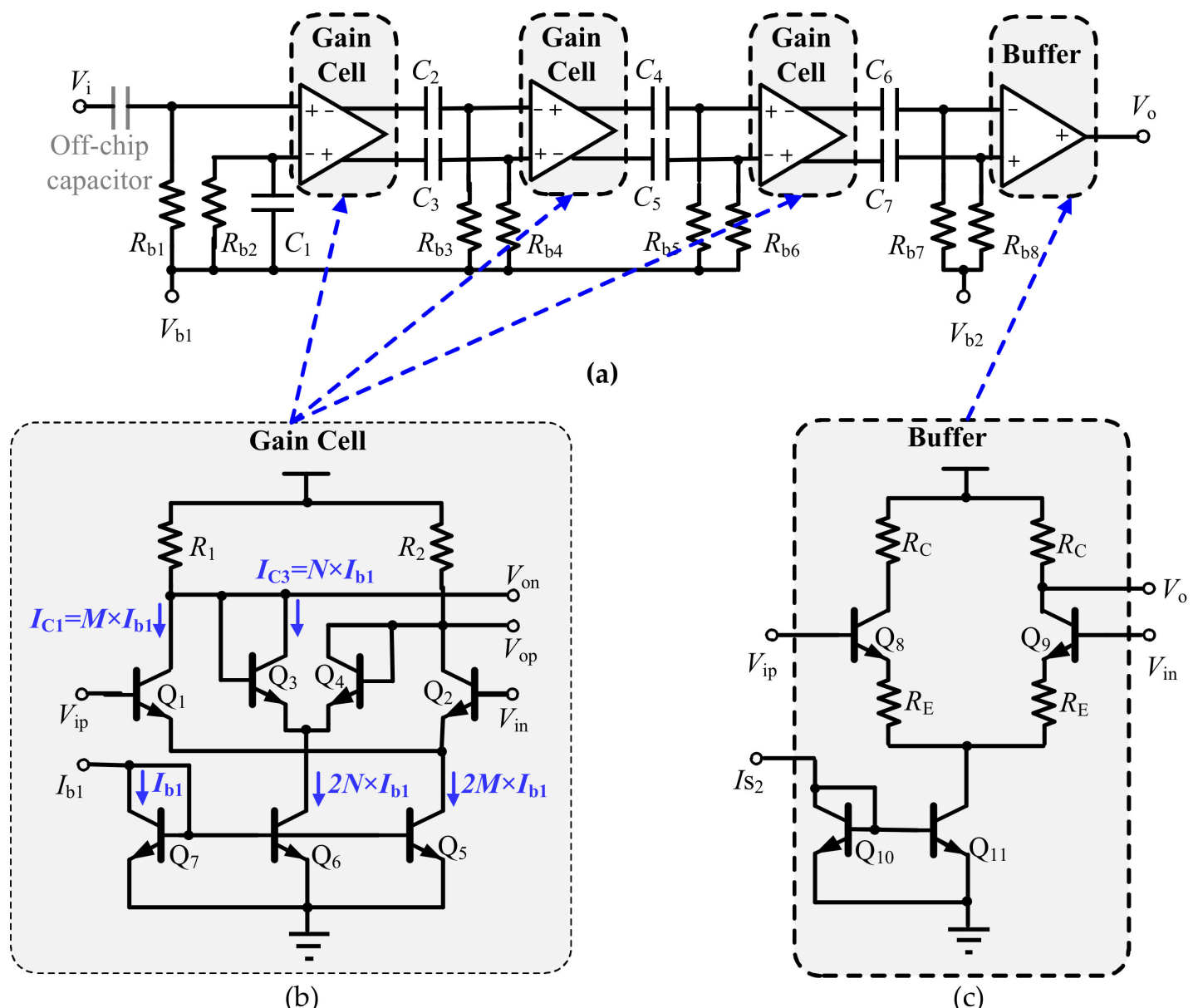

(b)

(c)

Figure 5. The proposed 4-stage cryogenic amplifier: (a) The amplifier topology; (b) Temperatureinsensitive gain cell; (c) Output buffer stage.

\section{The Temperature-Insensitive Gain Cell Design}

As mentioned before, bipolar transistors are sensitive to the temperature and its gain will change noticeable across a large temperature range. To solve this issue, a commonemitter gain cell topology with an emitter degeneration resistor can be used, as shown in Figure 6a. When the product of transistor $\mathrm{Q}_{1}$ transconductance and resistor $g_{\mathrm{m} 1} R_{\mathrm{E}}$ is significantly larger than 1 , the voltage gain of this cell is equal to the resistor ratio of $R_{\mathrm{C}} / R_{\mathrm{E}}$, which is insensitive to temperature variation. However, it should be noticed that, different to the thermal noise, the transistor shot noise does not scale with the temperature, and this gain cell suffers from large shot noise issues in the cryogenic environment. As a result, this emitter degeneration topology is not suitable for the input stage of the cryogenic amplifier, and it is employed as the output buffer with the merits of temperature-insensitive gain in this design, as shown in Figure 5c.

Figure $6 \mathrm{~b}$ shows another gain-cell topology, in which the conventional diode-connected transistors, $Q_{3}$ and $Q_{4}$, are realized as the load. As to be shown shortly, its gain is defined by the ratio of transistor $\mathrm{Q}_{1}$ and $\mathrm{Q}_{3}$ transconductances, i.e., $g_{\mathrm{m} 1}$ and $g_{\mathrm{m} 3}$. For proper biasing, the current mirror with PNP transistor $Q_{5}, Q_{6}$ and $Q_{10}$ is used to sink currents.

To illustrate the working mechanism of the gain cell in Figure $6 \mathrm{~b}$, for cascading the amplifier design and analysis convenience, taking into account the Miller capacitor and loading effects, Figure 7 shows the simplified single-ended equivalent circuit and its small signal model. Supposing the biasing current through $\mathrm{Q}_{7}$ is $I_{\mathrm{b}}$, with a proper current mirror size ratio, the collector currents through transistors $\mathrm{Q}_{1}$ and $\mathrm{Q}_{5}$ are $M \times I_{\mathrm{b}}$ and $(M-N) \times I_{\mathrm{b}}$, respectively. As a result, the current through transistor $\mathrm{Q}_{3}$ is equal to $N \times I_{\mathrm{b}}$. 


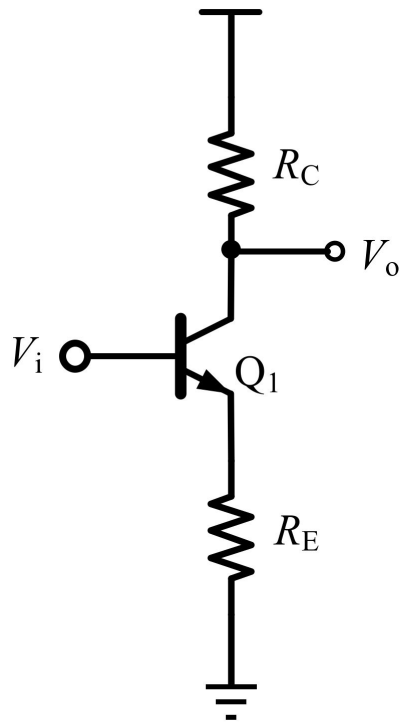

(a)

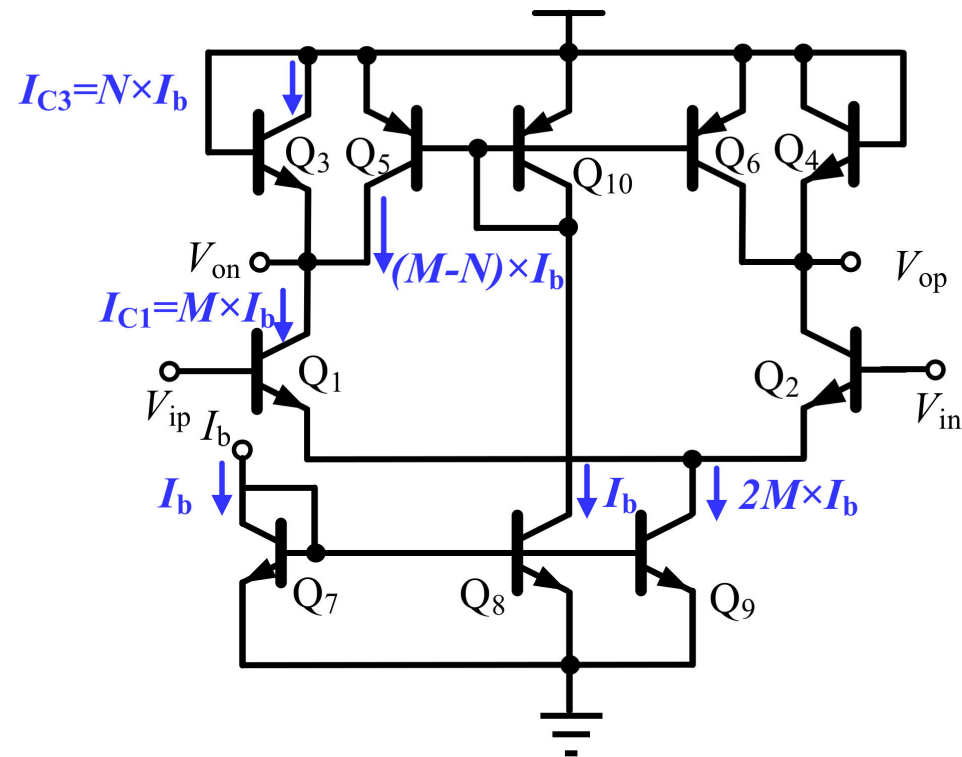

(b)

Figure 6. Schematic of (a) the typical common emitter gain cell with emitter degeneration; (b) the gain cell with the conventional diode-connected transistor load.
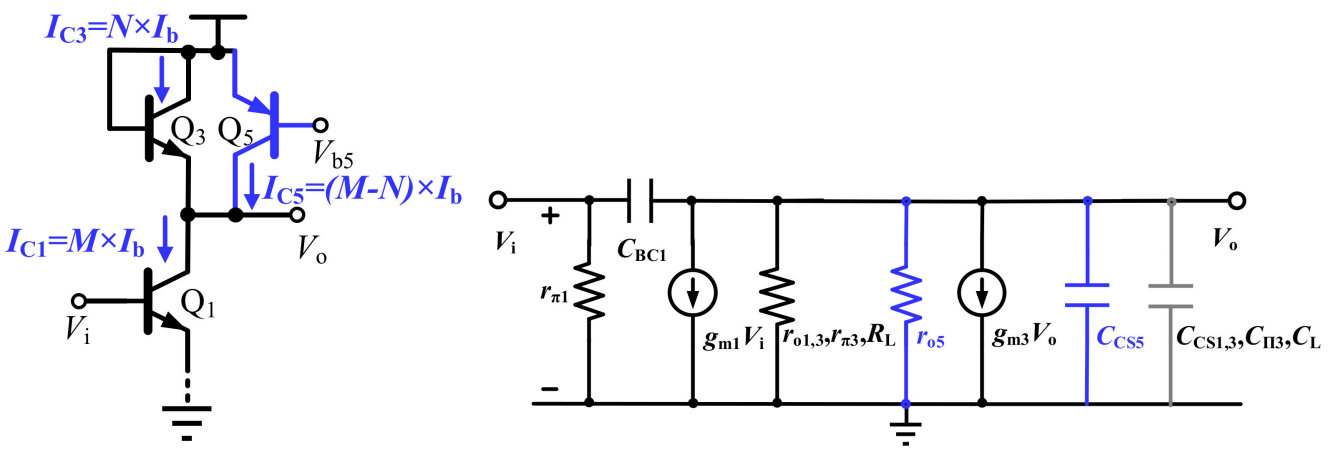

Figure 7. The simplified single-ended equivalent circuit and small signal model of the conventional gain cell.

The gain of the circuit is derived as follows:

$$
A_{v}=\frac{V_{o}}{V_{i}}=g_{m 1}\left(\frac{1}{g_{m 3}}\left\|r_{o 5}\right\| r_{o 1}\left\|r_{\pi 3}\right\| r_{o 3} \| R_{\mathrm{L}}\right)
$$

where $r_{\mathrm{o} 1,3,5}$ and $r_{\pi 1,3}$ are the output and input resistance of the transistors, respectively, and $R_{\mathrm{L}}$ is the input resistance of the transistor from the following gain stage. By definition, transconductance of the bipolar transistor $Q_{3}$ is given by

$$
g_{m 3}=M \times I_{\mathrm{b}} \frac{q}{k T}
$$

Equation (2) reveals the transistor transconductance strongly depends on its biasing current and operating temperature $T$. When the biasing current increases or the operating temperature decreases, $g_{\mathrm{m} 3}$ of the load transistor $\mathrm{Q}_{3}$ consequently increases. If the following condition is satisfied:

$$
\frac{1}{M \times I_{\mathrm{b}} \frac{q}{k T}}<<r_{05}\left\|r_{01}\right\| r_{\pi 3}\left\|r_{03}\right\| R_{\mathrm{L}}
$$


$1 / g_{m 3}$ would dominate the load impedance, and the gain of the circuit can be rewritten as follows:

$$
A_{v} \approx g_{m 1} \frac{1}{g_{m 3}}=\frac{M \times I_{\mathrm{b}} \frac{q}{k T}}{N \times I_{\mathrm{b}} \frac{q}{k T}}=\frac{M}{N}
$$

Accordingly, $A_{v}$ is equal to the current mirror ratio of $M / N$, and is not affected by the particular value of the biasing current $I_{\mathrm{b}}$ or temperature $T$. In other words, the gain performance of this gain cell becomes insensitive to the temperature.

The bandwidth is also derived as follows:

$$
\mathrm{BW}_{-3 \mathrm{~dB}} \approx \frac{1}{2 \pi \frac{1}{g_{m 3}}\left(C_{\mathrm{CS} 1}+C_{\pi 3}+C_{\mathrm{CS} 3}+C_{\mathrm{CS} 5}+C_{\mathrm{L}}+\left(1+\frac{1}{A_{v}}\right) C_{\mathrm{BC} 1}\right)} \approx \frac{q}{2 \pi k T} \frac{N \times I_{\mathrm{b}}}{\left(C_{\mathrm{CS} 1}+C_{\pi 3}+C_{\mathrm{CS} 3}+C_{\mathrm{CS} 5}+C_{\mathrm{L}}+C_{\mathrm{BC} 1}\right)}
$$

where $C_{\mathrm{CS} 1,3,5}$ and $C_{\pi 3}$ are the output and input parasitic capacitance of the transistors, respectively. Additionally, $C_{\mathrm{BC} 1}$ is the Miller capacitance of the transistors $Q_{1}$, and $C_{\mathrm{L}}$ includes the capacitance from Miller effect and input capacitance of the following gain stage. However, due to the large parasitic capacitance $C_{\mathrm{CS} 5}$ contributed by the PNP transistors $Q_{5}$, the bandwidth of the gain cell is reduced, as to be shown shortly.

Considering the above-mentioned issues, the diode-connected transistors $Q_{3,4}$ are folded in this design, leading to the proposed temperature-insensitive gain cell, as shown in Figure 5b. Different from the conventional diode-connected gain cell topology, correct DC paths are established by the resistor $R_{1,2}$ and the tail current sources $Q_{5,6}$. With a biasing current $I_{b 1}$ and a proper current-mirror ratio, the currents through transistor $\mathrm{Q}_{1}$ and $\mathrm{Q}_{3}$ are $M \times I_{\mathrm{b} 1}$ and $N \times I_{\mathrm{b} 1}$, respectively. In this way, the PNP transistors in Figure $6 \mathrm{~b}$ can be removed. For calculation convenience, Figure 8 shows the equivalent small signal model of the proposed gain cell.
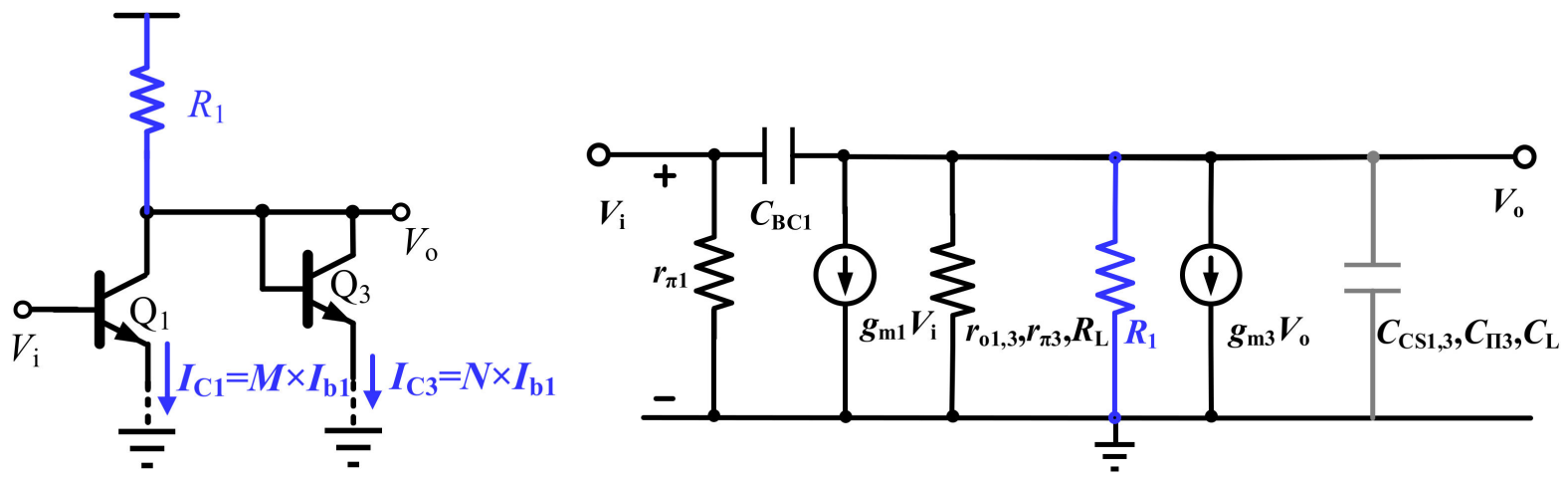

Figure 8. The simplified single-ended equivalent circuit and small signal model of the proposed gain cell with folded diode-connected load.

Similar to the theoretical derivations above, the gain and the bandwidth of the proposed gain cell are given by

$$
\begin{gathered}
A_{v}=g_{m 1}\left(\frac{1}{g_{m 3}}\left\|R_{1}\right\| r_{o 1}\left\|r_{\pi 3}\right\| r_{o 3} \| R_{\mathrm{L}}\right) \approx g_{m 1} \frac{1}{g_{m 3}}=\frac{M}{N} \\
\mathrm{BW}_{-3 \mathrm{~dB}} \approx \frac{q}{2 \pi k T} \frac{N \times I_{\mathrm{b} 1}}{\left(C_{\mathrm{CS} 1}+C_{\pi 3}+C_{\mathrm{CS} 3}+C_{\mathrm{L}}+C_{\mathrm{BC} 1}\right)}
\end{gathered}
$$

Note that the value of $R_{1,2}$ is chosen to be significantly larger than $1 / g_{\mathrm{m} 3,4}$, and it is realized by the ploy resistor with low temperature coefficient [26,27]. With simulations, it is proved that the amplifier bandwidth and gain change are slightly even with $\pm 35 \%$ resistance variation. Clearly, due to the good device tracking feature of NPN transistors, the voltage gain $A_{\mathrm{v}}$ of the proposed gain cell is determined only by the current mirror ratio $M / N$, which is three in this design. Equation (6) is valid both at room and cryogenic temperature when the transistor $Q_{3}$ transconductance dominants the load impedance, and the gain cell can achieve a constant gain at cryogenic temperature. It avoids the need of an accurate cryogenic device model during the design process.

Figure 9 compares the frequency responses of the conventional diode-connected gain cell and the proposed folded diode-connected gain cell at room temperature. Clearly, with $\mathrm{M} / \mathrm{N}$ ratio of 
three, both gain cells achieve a gain of $9.5 \mathrm{~dB}$, which agrees well with the theoretical calculation results. With the benefit of removing the large PNP transistor parasitic capacitance, the proposed folded diode-connected gain cell exhibits a higher bandwidth than the conventional cell with the same biasing current of $60-\mu \mathrm{A}$.

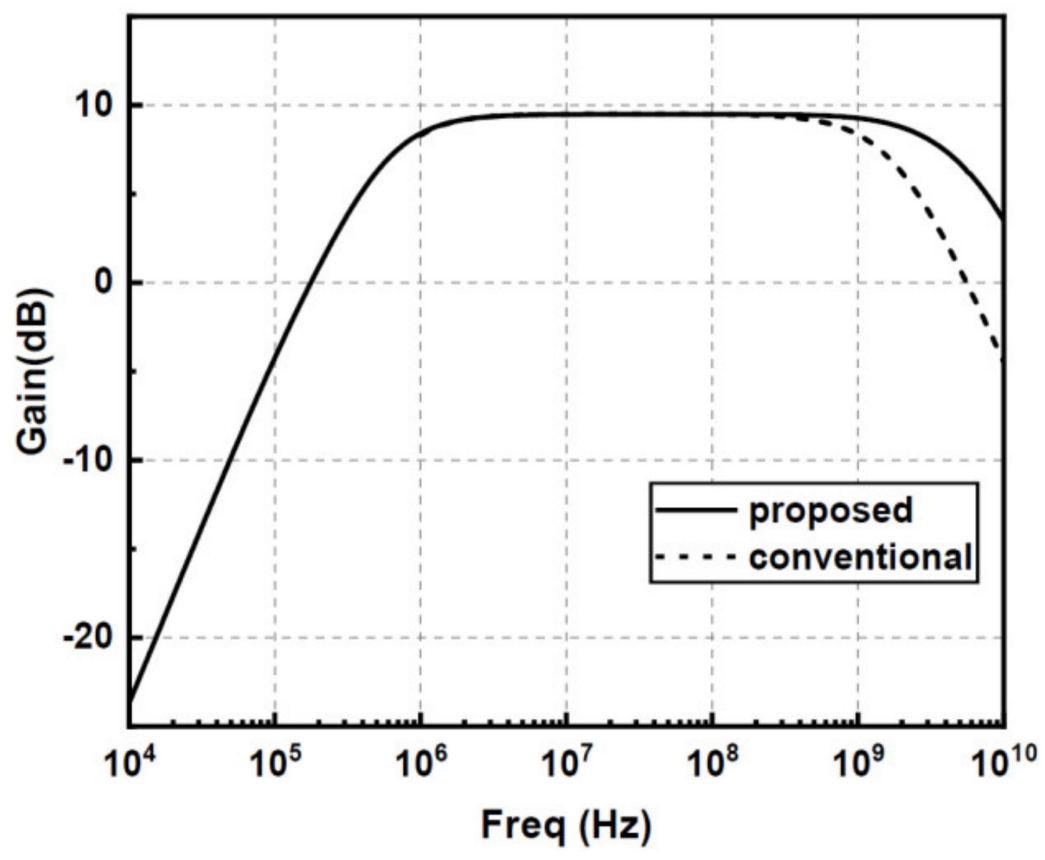

Figure 9. The simulated frequency response results of the proposed and the conventional gain cell.

As the valid temperature range for the commercial model of the SiGe process is from -40 to $120{ }^{\circ} \mathrm{C}$, to further illustrate the working mechanism of the proposed gain cell with different temperature and biasing current, in this design the amplifier gain performance at $100 \mathrm{MHz}$ is simulated with the above temperature range with a biasing current $I_{\mathrm{b} 1}$ ranging from 40 to $80 \mu \mathrm{A}$, as shown in Figure 10.

As indicated in Figure 10, at a particular temperature, the gain of the amplifier gradually approaches the saturation gain value of about $9.5 \mathrm{~dB}$ as the biasing current $I_{\mathrm{b} 1}$ increases. On the other hand, as the operating temperature decreases, the gain cell can reach a saturation value with a smaller biasing current $I_{\mathrm{b} 1}$. The transistor transconductance increases substantially when the temperature drops to a cryogenic temperature, even with a smaller biasing current [26]. Therefore, the transistor $\mathrm{Q}_{3}$ transconductance still dominates the load impedance even at cryogenic temperature, allowing the amplifier to achieve a saturation gain value.

The results can be understood and justified as follows: by increasing the biasing current or reducing operating temperature $T$, the transistor transconductance increases and dominates the load impedance; therefore, the gain reaches the saturation gain value close to the ratio $M / N$ of three, which is not affected by temperature variation. Therefore, the effectiveness of Equation (6) is proven, and the saturated gain performance of the gain becomes insensitive to temperature variation. With the above observations, it can be predicted that the biasing current required to achieve the saturated gain value can be reduced substantially at a cryogenic temperature.

Due to the lack of a cryogenic device model, for amplifier noise optimization, the noise simulation was undertaken at room temperature, showing that the equivalent integrated input referred noise amplitude within the passband from $1 \mathrm{MHz}$ to $1 \mathrm{GHz}$ is less than $70 \mu \mathrm{V}$. This input referred noise amplitude is significantly smaller than typical SNSPD input signal amplitude. As highlighted by [28], the transistor and the amplifier noise decreased substantially at cryogenic temperature, and it can be predicted that the cryogenic amplifier has good noise performance for SNSPDreadout applications. 


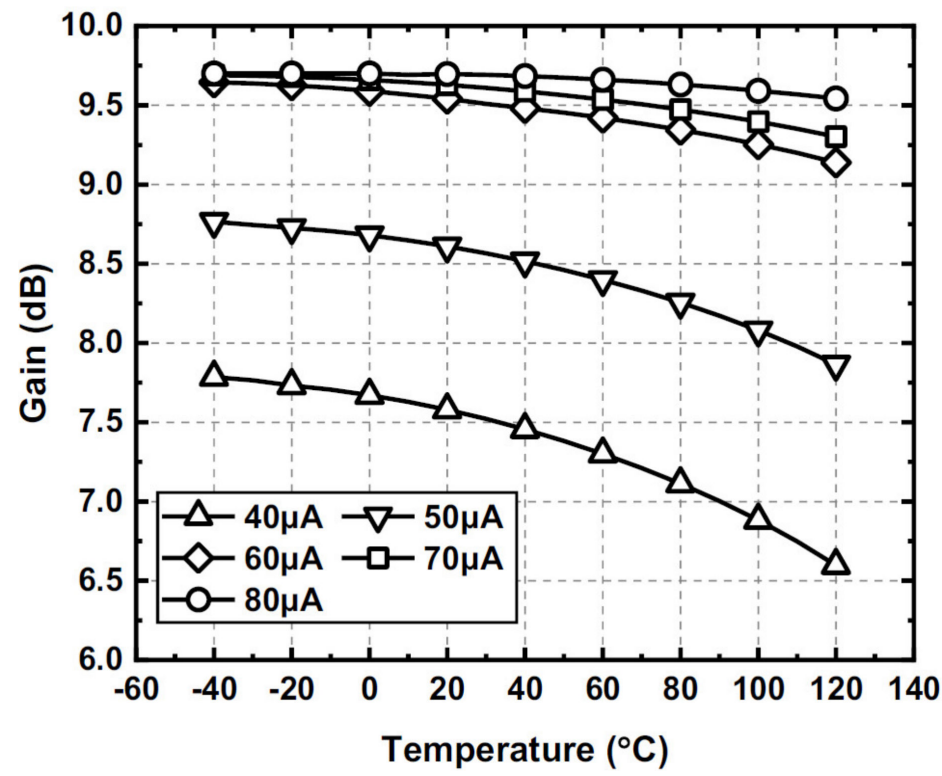

Figure 10. The simulated gain at $100 \mathrm{MHz}$ of the proposed gain cell with different operating temperatures and biasing currents.

\section{Implementation, Simulation and Measurement Results}

With the benefit of high integration and low cost, the proposed amplifier is fully integrated in a single chip and fabricated with a $0.13 \mu \mathrm{m}$ SiGe BiCMOS process, which provides a high performance NPN transistor with a peak $f_{\mathrm{T}}$ of $210 \mathrm{GHz}$, poly resistors, metal-insulator-metal (MIM) capacitors and seven metal layers. Figure 11a shows the realized PCB test board, and the SMA connectors are used for input and output ports. Figure $11 \mathrm{~b}$ shows the amplifier die microphotograph and the chip area is about $1 \mathrm{~mm} \times 0.5 \mathrm{~mm}$. The amplifier measurements were undertaken at room and cryogenic temperatures, respectively.

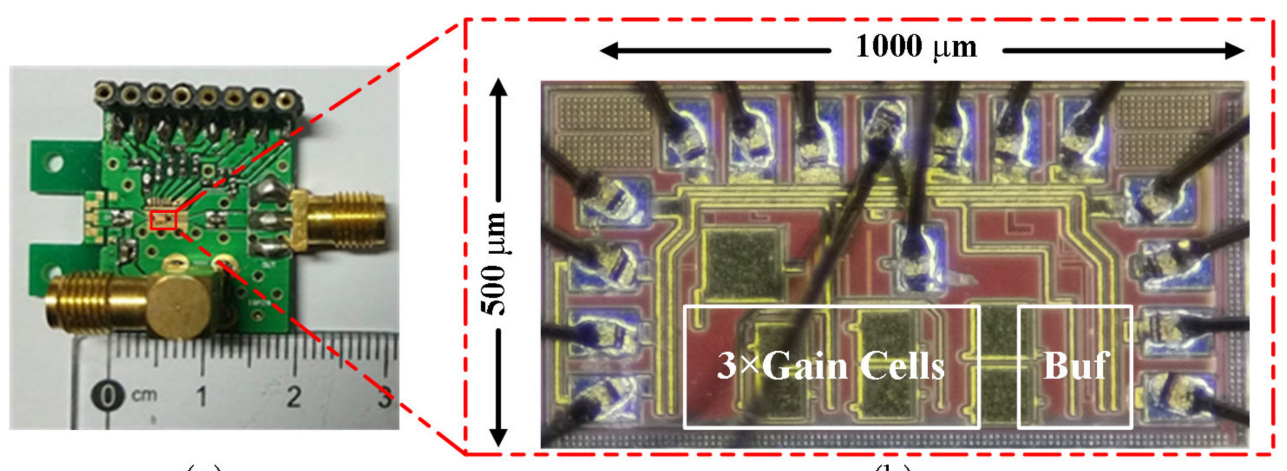

(a)

(b)

Figure 11. The photograph of PCB test board and the cryogenic amplifier chip: (a) The PCB board; (b) Chip microphotograph.

\subsection{Room Temperature Performance}

With the biasing voltage $V_{\mathrm{B} 1}$ and $V_{\mathrm{B} 2}$ of $1.5 \mathrm{~V}$, the amplifier is biased with $I_{\mathrm{b} 1}$ and $I_{\mathrm{b} 2}$ of 60 and $150 \mu \mathrm{A}$, respectively, and consumes a current of $4.5 \mathrm{~mA}$ from a $1.8 \mathrm{~V}$ supply voltage. Measured with a network analyzer, Figure 12 shows the measured gain at $300 \mathrm{~K}$ room temperature. As indicated, the amplifier achieved a gain of $26 \mathrm{~dB}$ with bandwidth over $1 \mathrm{GHz}$. Moreover, the amplifier measurement results are compared with the simulation results under the same biasing condition, showing good agreement with each other. Note that due to the bonding wire parasitic inductance, some gain ripples were introduced at a high frequency. 


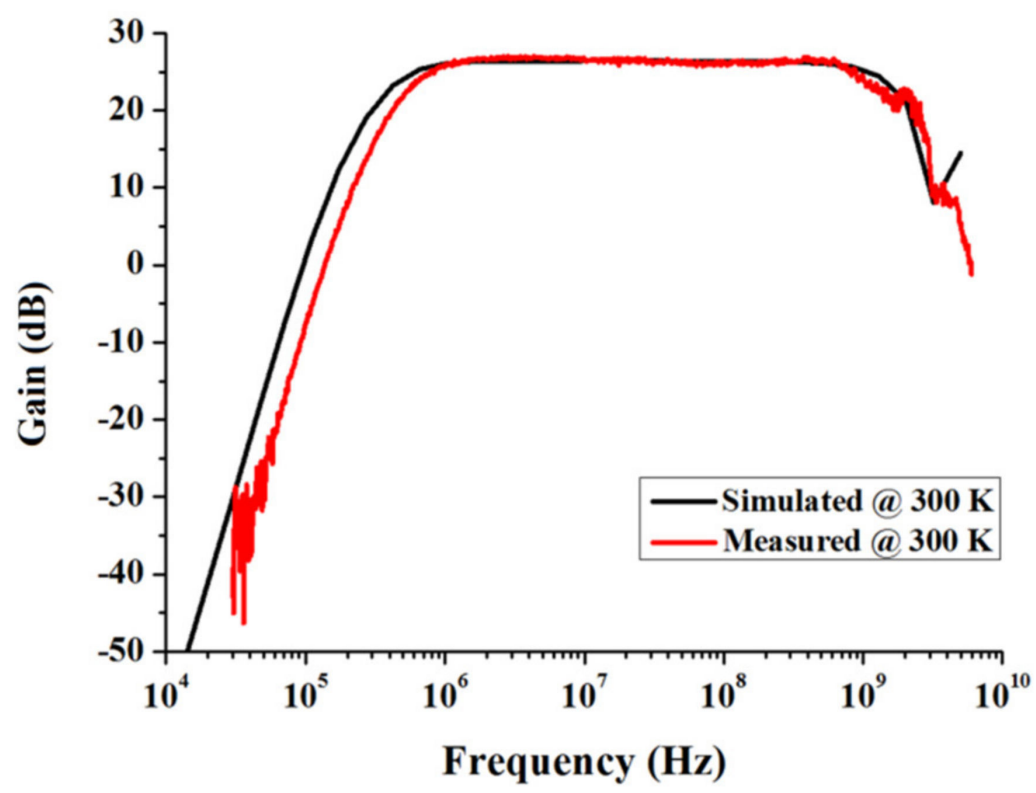

Figure 12. Simulated and measured gain of the amplifier at $300 \mathrm{~K}$.

\subsection{Cryogenic Performance}

Figure 13 shows the measurement setup at cryogenic temperature. With the help of the special Dewar containing the liquid helium, the amplifier was cooled down to the specific temperature of $4.2 \mathrm{~K}$. Note that two chips are tested in this work and their results are quite close to each other. For clearer illustration, only one of the chip results are shown in the following parts.

With the same biasing voltage $V_{\mathrm{B} 1}$ and $V_{\mathrm{B} 2}$ of $1.5 \mathrm{~V}$ at $300 \mathrm{~K}$, Figure 14 shows the measured gain with a biasing current $I_{\mathrm{b} 1}$ ranging from 1 to $12 \mu \mathrm{A}$, while $I_{\mathrm{b} 2}$ is set to $50 \mu \mathrm{A}$. As illustrated, the amplifier gain improved with increased biasing current $I_{\mathrm{b} 1}$. When the biasing current $I_{\mathrm{b} 1}$ was larger than $9 \mu \mathrm{A}$, the amplifier gain was saturated to $26 \mathrm{~dB}$. Compared with Figure 12, it can be seen that the amplifier achieved the same saturated gain at $4.2 \mathrm{~K}$ and $300 \mathrm{~K}$. It also indicates that, when the temperature decreased dramatically to $4.2 \mathrm{~K}$, with a lower biasing current, the amplifier still achieved a predicable saturated gain even without an accurate cryogenic device model. With such advantages, the cryogenic amplifier consumes $1.8 \mathrm{~mW}$ with $I_{\mathrm{b} 1}=9 \mu \mathrm{A}$, which is only $22 \%$ of the amplifier power consumption at room temperature. These results agree well with the theoretical analysis, simulation analysis and prediction in Section 3, proving the effectiveness of the proposed temperature-insensitive gain cell.

As the noise performance of the cryogenic amplifier is excellent, the measurement accuracy is severely limited by the conversional noise figure (NF) measurement method $[26,29]$. Because of the limitation of the test equipment, it is reasonable to apply a sinusoidal stimulate signal for evaluating the amplifier noise performance, as what has been performed in [19], instead of using the cold attenuator NF test setup [26,30]. To evaluate such performance, with a $1 \mathrm{GHz}$ input sinusoid signal of $-60 \mathrm{dBm}$, which is the minimum signal provided by the analogue signal generator, Figure 15 shows the amplified output signal. As indicated, even when taking into account the supply noise, the noise of the measurement equipment and unwanted interferences, the output signal is clear and distinguishable at $4.2 \mathrm{~K}$. With FFT calculation on the output transient signal, the calculated SNR is about $17 \mathrm{~dB}$, proving that the amplifier has good SNR performance. When the input sinusoid signal increased by ten times, i.e., $-50 \mathrm{dBm}$, Figure 16 shows the amplified output signal. The output signal amplitude is about $101 \mathrm{mV}$, indicating a gain compression of around $1 \mathrm{~dB}$. It proves that the amplifier linearity meets the SNSPD readout requirements.

Table 1 summarizes state-of-the-art cryogenic amplifiers operating within a several $\mathrm{GHz}$ frequency range $[19,26,27,30,31]$. For performance comparison, a figures of merit (FOM) defined in [19] is utilized

$$
F O M=\frac{\text { Gain } \cdot \mathrm{BW}(\mathrm{GHz})}{\mathrm{P}_{\text {diss }}(m W) \cdot \operatorname{Area}\left(\mathrm{mm}^{2}\right)}
$$


Moreover, a new figure of merit $\left(F O M_{2}\right)$ adding the impact of process transition frequency $f_{\mathrm{T}}$ is defined for the performance comparison, which is calculated as:

$$
F O M_{2}=\frac{1}{\mathrm{P}_{\text {diss }}(m W) \cdot \operatorname{Area}\left(\mathrm{mm}^{2}\right)} \frac{\mathrm{Gain} \cdot \mathrm{BW}(\mathrm{GHz})}{f_{\mathrm{T}}(\mathrm{GHz})}
$$

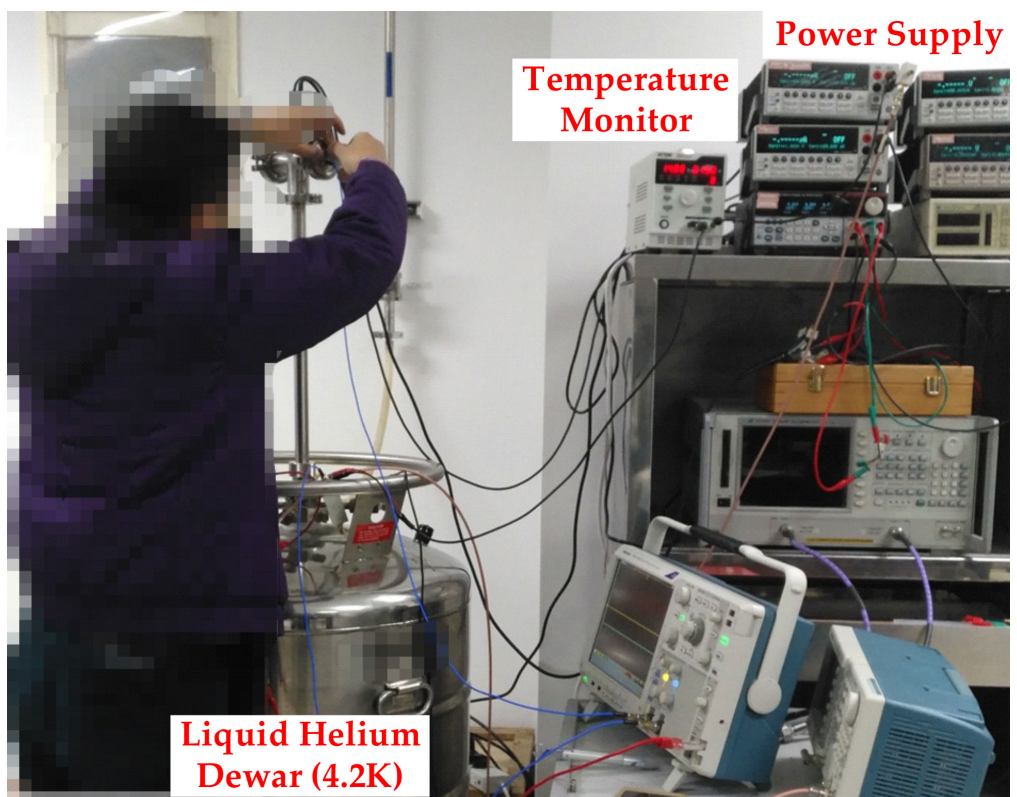

Figure 13. The measurement setup at cryogenic temperature.

As indicated, the proposed amplifier shows competitive $F O M$ and $F O M_{2}$ values. Compared to the other cryogenic amplifiers, with the lowest power consumption, this work achieves good gain and bandwidth performance. To achieve optimum cryogenic amplifier performance, in $[19,30]$ the amplifiers need to be tuned carefully. Different from these solutions, in this paper, without complex biasing tuning, the proposed amplifier achieved a robust saturated gain value, which is insensitive to temperature. Moreover, compared to [19], its power consumption is reduced by $58 \%$.

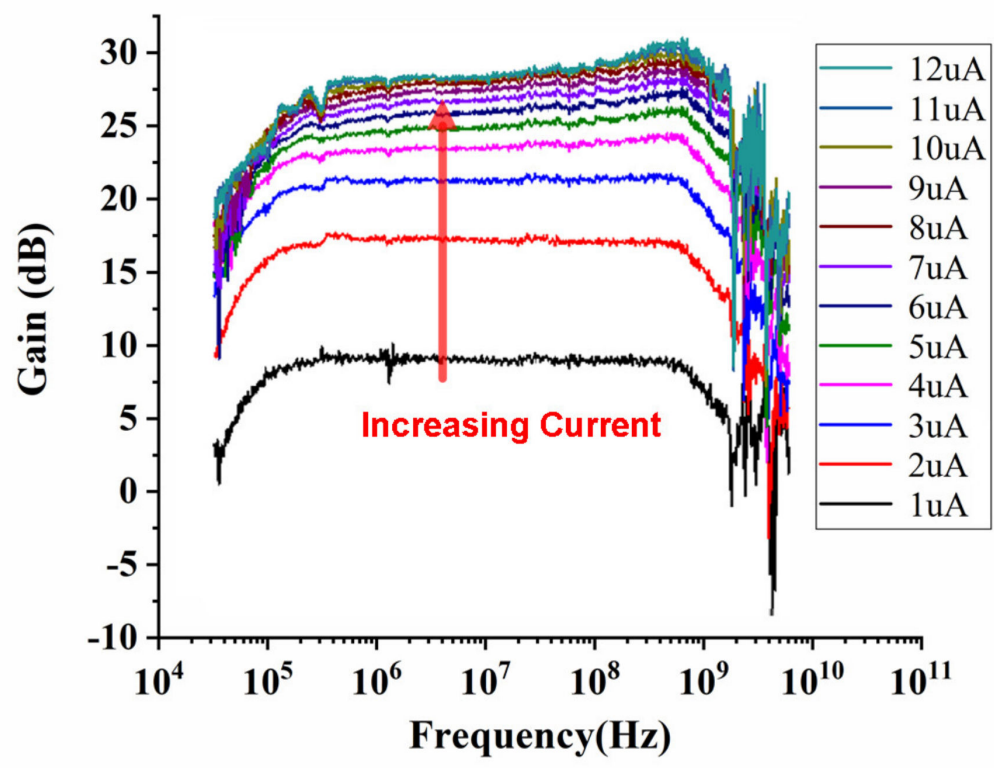

Figure 14. Measured gain with different biasing current $I_{\mathrm{b} 1}$ ranging from 1 to $12 \mu \mathrm{A}$ at $4.2 \mathrm{~K}$. 


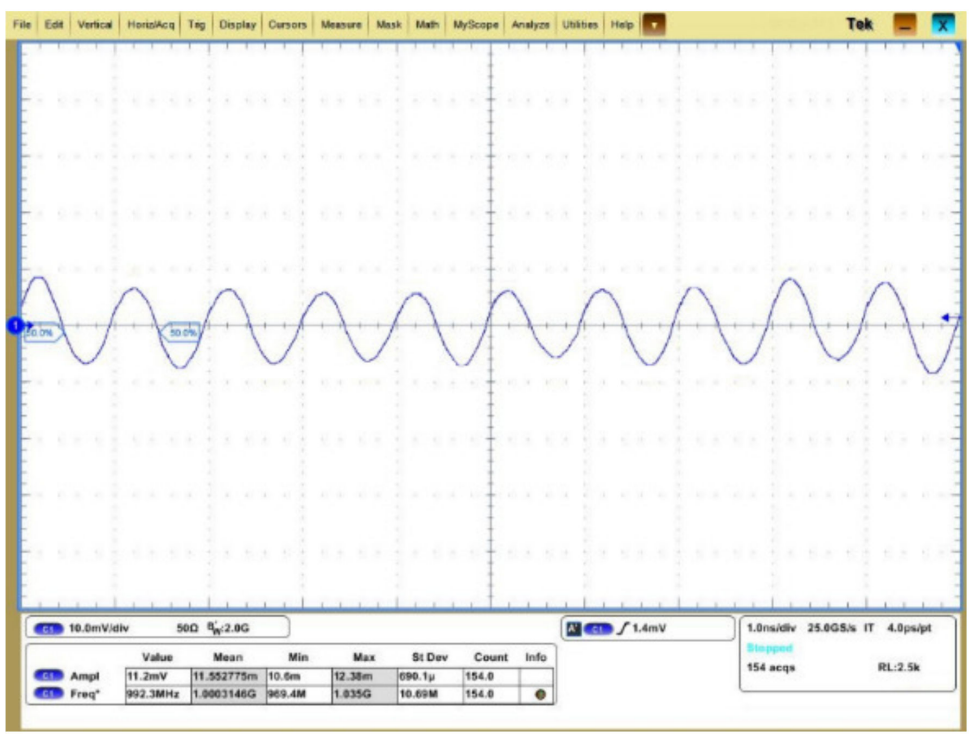

Figure 15. 11.2-mV 1-GHz output sinusoid signal at $4.2 \mathrm{~K}$.

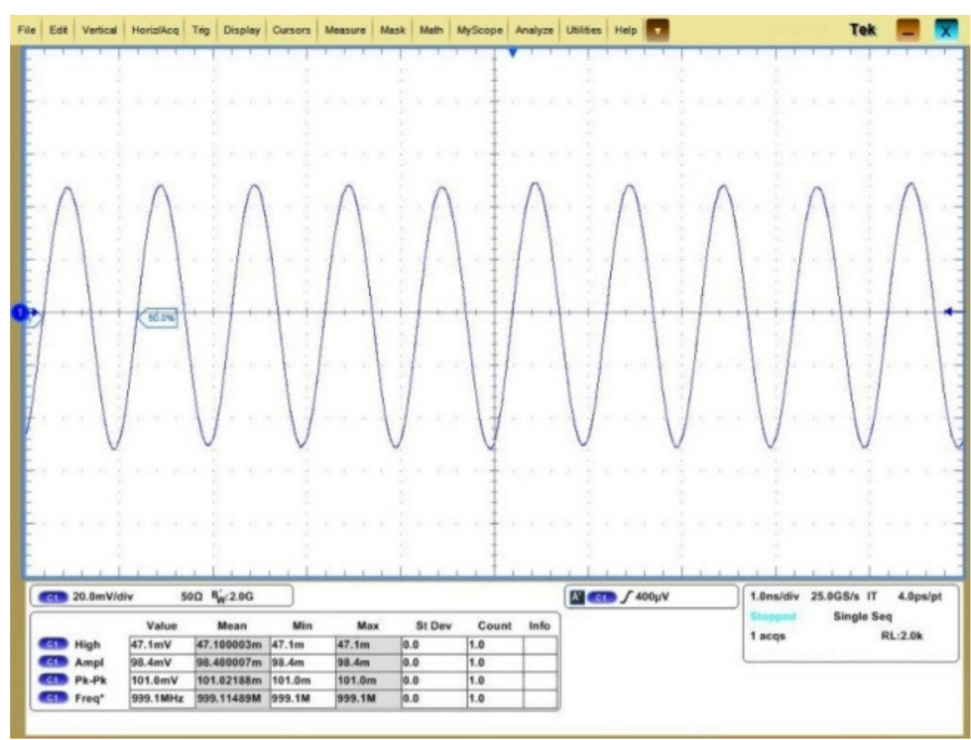

Figure 16. 101-mV 1-GHz output sinusoid signal at $4.2 \mathrm{~K}$.

Table 1. Comparison with state-of-the-art cryogenic amplifiers.

\begin{tabular}{|c|c|c|c|c|c|c|}
\hline References & [19] & [26] & [27] & [30] & [31] & This Work \\
\hline Technology & $\mathrm{SiGe}$ & SiGe & $\mathrm{SiGe}$ & $\mathrm{SiGe}$ & GaAs & SiGe \\
\hline Gain (dB) & 23 & 29.6 & 22 & 10 & 15 & 26 \\
\hline Bandwidth (GHz) & 3.4 & 4.9 & 2.7 & 3.4 & 1.5 & 1 \\
\hline $\begin{array}{l}\text { Power Dissipation } \\
\qquad(\mathrm{mW})\end{array}$ & 4.3 & 20 & 32 & 13.5 & 2.5 & 1.8 \\
\hline Area $\left(\mathrm{mm}^{2}\right)$ & 0.5 & 0.3 & 0.6 & 0.3 & $\mathrm{~N} / \mathrm{A}$ & 0.5 \\
\hline FOM & 22 & 25 & 1.8 & 2.6 & $\mathrm{~N} / \mathrm{A}$ & 22 \\
\hline$f_{\mathrm{T}}(\mathrm{GHz})$ & 210 & 210 & 200 & 120 & $\mathrm{~N} / \mathrm{A}$ & 210 \\
\hline FOM2 & 0.105 & 0.119 & 0.009 & 0.022 & $\mathrm{~N} / \mathrm{A}$ & 0.105 \\
\hline
\end{tabular}




\section{Conclusions}

To solve the lack of the accurate cryogenic device model issues and to increase robustness, by introducing a folded diode-connected transistor load, this paper proposes a temperature-insensitive wideband cryogenic amplifier for SNSPD readout applications. With a good device-tracking feature, the theoretical derivations, simulation and test results prove that the gain cell has a stable gain performance across a large temperature range. The amplifier achieves a gain of $26 \mathrm{~dB}$ with a bandwidth over $1 \mathrm{GHz}$ at $4.2 \mathrm{~K}$, consuming only $1.8 \mathrm{~mW}$ from a supply voltage of $1.8 \mathrm{~V}$. With a $0.13 \mu \mathrm{m}$ SiGe BiCMOS process, the chip area is only $0.5 \mathrm{~mm}^{2}$.

Author Contributions: Conceptualization, X.N., L.L., D.W. and X.W.; Investigation, X.N. and L.L.; Writing-original draft preparation X.N., L.L. and X.W.; Writing-review and editing, X.W. All authors have read and agreed to the published version of the manuscript.

Funding: This work was supported in part by the National Nature Science Foundation of China under Grant 61306030 and Grant 61674037, and in part by the Natural Science Foundation of Jiangsu Province of China under Grant BK20180368.

Institutional Review Board Statement: Not applicable.

Informed Consent Statement: Not applicable.

Data Availability Statement: Not applicable.

Acknowledgments: The authors would like to express their appreciation to Long He, for assistance with investigation work.

Conflicts of Interest: The authors declare no conflict of interest.

\section{References}

1. Jia, T.; Kang, L.; Zhang, L.; Zhao, Q.; Gu, M.; Qiu, J.; Chen, J.; Jin, B. Doped niobium superconducting nanowire single-photon detectors. Appl. Phys. A 2014, 116, 991-995. [CrossRef]

2. Natarajan, C.M.; Tanner, M.; Hadfield, R. Superconducting nanowire single-photon detectors: Physics and applications. Supercond. Sci. Technol. 2012, 25, 63001. [CrossRef]

3. Takesue, H.; Nam, S.; Zhang, Q.; Hadfield, R.; Honjo, T.; Tamaki, K.; Yamamoto, Y. Quantum key distribution over a 40-db channel loss using superconducting sin-gle-photon detectors. Nat. Photonics 2007, 1, 343-348. [CrossRef]

4. $\quad$ Takesue, H.; Dyer, S.D.; Stevens, M.J.; Verma, V.; Mirin, R.P.; Nam, S.W. Quantum tel-eportation over 100 km of fiber using highly efficient superconducting nanowire sin-gle-photon detectors. Optica 2015, 2, 832-835. [CrossRef]

5. McCarthy, A.; Krichel, N.J.; Gemmell, N.R.; Ren, X.; Tanner, M.; Dorenbos, S.N.; Zwiller, V.; Hadfield, R.; Buller, G. Kilometerrange, high resolution depth imaging via $1560 \mathrm{~nm}$ wavelength single-photon detection. Opt. Express 2013, 21, 8904-8915. [CrossRef]

6. Niwa, K.; Numata, T.; Hattori, K.; Fukuda, D. Few-photon color imaging using ener-gy-dispersive superconducting transitionedge sensor spectrometry. Sci. Rep. 2017, 7, 45660. [CrossRef]

7. Geissbuehler, S.; Bocchio, N.L.; Dellagiacoma, C.; Berclaz, C.; Leutenegger, M.; Lasser, T. Mapping molecular statistics with balanced super-resolution optical fluctuation imaging (bSOFI). Opt. Nanoscopy 2012, 1, 4. [CrossRef]

8. Nichols, A.J.; Evans, C.L. Video-rate Scanning Confocal Microscopy and Microendoscopy. J. Vis. Exp. 2011, 56, e3252. [CrossRef]

9. Crespi, A.; Lobino, M.; Matthews, J.; Politi, A.; Neal, C.R.; Ramponi, R.; Osellame, R.; O’Brien, J. Measuring protein concentration with entangled photons. Appl. Phys. Lett. 2012, 100, 233704. [CrossRef]

10. Degen, C.; Reinhard, F.; Cappellaro, P. Quantum sensing. Rev. Mod. Phys. 2016, 89, 35002. [CrossRef]

11. Wuensch, S.; Bruch, D.; Crocoll, E.; Leuther, A.; Kallfass, I.; Siegel, M. Development of Monolithic Microwave Integrated Amplifiers as Readout for Detectors at 4.2 K. IEEE Trans. Appl. Supercond. 2011, 21, 302-305. [CrossRef]

12. Lotfi, S.; Roshani, S.; Roshani, S.; Shirzadian Gilan, M. A planner Doherty power amplifier with harmonic suppression with open and short ended stubs. Frequenz 2021, 30. [CrossRef]

13. Hookari, M.; Roshani, S.; Roshani, S. High-efficiency balanced power amplifier using miniaturized harmonics suppressed coupler. Int. J. RF Microw. 2020, 30, e22252. [CrossRef]

14. Gaggero, A.; Martini, F.; Mattioli, F.; Chiarello, F.; Cernansky, R.; Politi, A.; Leoni, R. Amplitude-multiplexed readout of single photon detectors based on superconducting nanowires. Optica 2019, 6, 823-828. [CrossRef]

15. Ravindran, P.; Cheng, R.; Tang, H.; Bardin, J.C. Active quenching of superconducting nanowire single photon detectors. Opt. Express 2020, 28, 4099-4114. [CrossRef]

16. Schleeh, J.; Wadefalk, N.; Nilsson, P.-Å.; Starski, J.P.; Grahn, J. Cryogenic Broadband Ultra-Low-Noise MMIC LNAs for Radio Astronomy Applications. IEEE Trans. Microw. Theory Tech. 2013, 61, 871-877. [CrossRef] 
17. Montazeri, S.; Bardin, J.C. A sub-milliwatt 4-8 GHz SiGe cryogenic low noise amplifier. In Proceedings of the 2017 IEEE MTT-S International Microwave Symposium (IMS), Honololu, HI, USA, 4-9 June 2017; pp. 160-163. [CrossRef]

18. Cha, E.; Wadefalk, N.; Moschetti, G.; Pourkabirian, A.; Stenarson, J.; Grahn, J. A 300- $\mu$ W Cryogenic HEMT LNA for Quantum Computing. In Proceedings of the 2020 IEEE MTT-S International Microwave Symposium (IMS2020), Los Angeles, CA, USA, 21-26 June 2020; pp. 1299-1302.

19. He, L.; Li, L.; Niu, X.; Haiyang, X.; Xie, M.; Wu, X.; Zhang, L.; Kang, L.; Wang, Z. A Low-Power, Inductorless Wideband Cryogenic Amplifier For Supercondcting Nanowire Single Photon Detector. IEEE Trans. Appl. Supercond. 2019, 29, 1-6.

20. Berggren, K.; Zhao, Q.; Abebe, N.; Chen, M.; Ravindran, P.; McCaughan, N.; Bardin, J. A Superconducting Nanowire can be Modeled by Using SPICE. Supercond. Sci. Technol. 2018, 31, 55010. [CrossRef]

21. Bardin, J.C.; Ravindran, P.; Chang, S.-W.; Kumar, R.; Stern, J.A.; Shaw, M.D.; Russell, D.S.; Farr, W.H. A high-speed cryogenic SiGe channel combiner IC for large photon-starved SNSPD arrays. In Proceedings of the 2013 IEEE Bipolar/BiCMOS Circuits and Technology Meeting (BCTM), Bordeaux, France, 30 September-3 October 2013.

22. Bardin, J.C.; Ravindran, P.; Chang, S.-W.; Mohamed, C.; Kumar, R.; Stern, J.A.; Shaw, M.D.; Russell, D.; Marsili, F.; Resta, G.; et al. Cryogenic SiGe integrated circuits for superconducting nanowire single photon detector readout. In Proceedings of the SPIE Sensing Technology + Applications, Baltimore, MD, USA, 5-9 May 2014; p. 911404.

23. Ramkaj, A.T.; Steyaert, M.S.J.; Tavernier, F. A 13.5-Gb/s 5-mV-Sensitivity 26.8-ps-CLK-OUT Delay Triple-Latch Feedforward Dynamic Comparator in 28-nm CMOS. IEEE Solid-State Circuits Lett. 2019, 2, 167-170. [CrossRef]

24. Bardin, J.; Weinreb, S. A 0.1-5 GHz cryogenic SiGe MMIC LNA. IEEE Microw. Wirel. Compon. Lett. 2009, 19, 407-409. [CrossRef]

25. Chang, S.-W.; Bardin, J. A Wideband cryogenic SiGe LNA MMIC with an average noise temperature of $2.8 \mathrm{~K}$ from 0.3-3 GHz. In Proceedings of the 2017 IEEE MTT-S International Microwave Symposium (IMS2017), Honolulu, HI, USA, 4-9 June 2017; pp. 157-159.

26. Weinreb, S.; Bardin, J.; Mani, H. Design of Cryogenic SiGe Low-Noise Amplifiers. IEEE Trans. Microw. Theory Tech. 2007, 55, 2306-2312. [CrossRef]

27. Russell, D.; Weinreb, S. Low-Power Very Low-Noise Cryogenic SiGe IF Amplifiers for Terahertz Mixer Receivers. IEEE Trans. Microw. Theory Tech. 2012, 60, 1641-1648. [CrossRef]

28. Bardin, J.C.; Weinreb, S. Experimental cryogenic modeling and noise of SiGe HBTs. Proceedings of 2008 IEEE MTT-S International Microwave Symposium (IMS2008), San Francisco, CA, USA, 15-20 June 2008; pp. 459-462.

29. Shiao, Y.S.J.; Huang, G.W.; Chiueh, T.H. A 4 GHz cryogenic amplifier in $0.18 \mu \mathrm{m}$ general purpose BiCMOS technology. Proceedings of 2014 Asia-Pacific Microwave Conference (APMC 2014), Sendai, Japan, 4-7 November 2014; pp. 1181-1183.

30. Fernandez, J. A noise-temperature measurement system using a cryogenic attenuator. TMO Prog. Rep. 1998, $15,42-135$.

31. Cahall, C.T.; Gauthier, D.J.; Kim, J. Cryogenic Amplifiers for a Superconducting Nanowire Single Photon Detector System. In Proceedings of the Conference on Lasers and Electro-Optics (CLEO), San Jose, CA, USA, 5-10 June 2016; pp. 5-10. 